\title{
Combined Neurofeedback and Heart Rate Variability Training for Individuals with Symptoms of Anxiety and Depression: A Retrospective Study
}

\author{
Elyse K. White ${ }^{1^{*}}$, Kayleah M. Groeneveld ${ }^{1}$, Rachel K. Tittle ${ }^{1}$, Nicholas A. Bolhuis ${ }^{1}$, Rachel E. \\ Martin $^{1}$, Timothy G. Royer ${ }^{2}$, and Majid Fotuhi, ${ }^{1,3}$ \\ ${ }^{1}$ Neurocore, Grand Rapids, Michigan, USA \\ ${ }^{2}$ Neurocore Pro, Grand Rapids, Michigan, USA \\ 3Johns Hopkins Medicine, Baltimore, Maryland, USA
}

\begin{tabular}{|c|c|}
\hline \multicolumn{2}{|c|}{ Abstract } \\
\hline $\begin{array}{l}\text { Introduction. Neurofeedback (NFB) and heart } \\
\text { nonpharmaceutical intervention strategies for anxiety anc } \\
\text { concurrent NFB and HRV (NFB+HRV) provides a viabl } \\
\text { measured by the Achenbach System of Empirically Base } \\
\text { children and adults with symptoms of anxiety and/or de } \\
\text { symptom rating, EEG, blood pressure, breathing pattern } \\
\text { Results. After NFB+HRV training, symptoms of anxiety } \\
\text { were reduced in children and adults. The majority of inc } \\
\text { or depression ( } 81.1 \% \text { ) experienced ASEBA improveme } \\
\text { changes in EEG, breathing rate, and HRV. For the } 16 \\
\text { diastolic blood pressure were significantly reduced. Cor } \\
\text { may provide an effective, nonpharmaceutical interventi } \\
\text { children and adults. Additionally, NFB+HRV training ma } \\
\text { and HRV. }\end{array}$ & $\begin{array}{l}\text { rate variability (HRV) training present promising, } \\
\text { d depression. This report is the first to address whether } \\
\text { e intervention for symptoms of anxiety and depression, } \\
\text { ed Assessment (ASEBA) questionnaire. Methods. } 183 \\
\text { epression underwent NFB+HRV training. Psychological } \\
\mathrm{n} \text {, and HRV were measured before and after treatment. } \\
\left(p<.001, d_{z}=1.42\right) \text { and depression }\left(p<.001, d_{z}=1.34\right) \\
\text { dividuals with pretreatment symptoms of anxiety }(82.8 \%) \\
\text { ents of clinical importance. There were also significant } \\
\text { individuals copresenting with hypertension, systolic and } \\
\text { nclusion. We present evidence that NFB+HRV training } \\
\text { ion to reduce symptoms of anxiety and depression in } \\
\text { ay improve EEG, blood pressure, resting breathing rate, }\end{array}$ \\
\hline $\begin{array}{l}\text { ion: White, E. K., Groeneveld, K. M., Tittle } \\
\text { ofeedback and heart rate variability trainin } \\
\text { oRegulation, } 4(1), 37-55 . \text { http://dx.doi.org/10. }\end{array}$ & $\begin{array}{l}\text { A., Martin, R. E., Royer, T. G., \& Fotuhi, M. (2017). Combined } \\
\text { symptoms of anxiety and depression: A retrospective study. }\end{array}$ \\
\hline $\begin{array}{l}\text { *Address correspondence to: Elyse K. White, PhD, Neurocore, } 201 \\
\text { Monroe Avenue NW, Suite 300, Grand Rapids, Ml 49503, USA. } \\
\text { Email: elyse.kw@neurocorecenters.com } \\
\text { Copyright: ( } 2 \text { 2017. White et al. This is an Open Access article } \\
\text { distributed under the terms of the Creative Commons Attribution } \\
\text { License (CC-BY). }\end{array}$ & $\begin{array}{l}\text { Edited by: } \\
\text { Rex L. Cannon, PhD, Neurogifted, Boynton Beach, Florida, USA } \\
\text { Reviewed by: } \\
\text { John Davis, PhD, McMaster University, Hamilton, Ontario, Canada } \\
\text { Randall Lyle, PhD, Mount Mercy University, Cedar Rapids, lowa, } \\
\text { USA }\end{array}$ \\
\hline
\end{tabular}

\section{Introduction}

Generalized anxiety disorder (GAD) and major depressive disorder (MDD) are affective psychological disorders that affect millions of Americans. These diseases cause considerable morbidity and mortality, as well as substantial private and public economic burden (Asselmann \& BeesdoBaum, 2015; Kessler et al., 2007; Richards, 2011).
Many people do not gain satisfactory results from pharmaceutical approaches such as anxiolytics or Selective Serotonin Reuptake Inhibitor medications, which furthermore cause substantial side effects (Abejuela \& Osser, 2016; Carvalho, Sharma, Brunoni, Vieta, \& Fava, 2016; Kirsch et al., 2008). Accordingly, nonpharmaceutical interventions to complement or replace treatment with psychoactive medications are of great importance. 
Psychotherapeutic approaches to treat anxiety and depression are effective; however, psychotherapy only partially reduces the disease burden, does not work for everyone, and people often relapse after the conclusion of treatment (Andrews, Issakidis, Sanderson, Corry, \& Lapsley, 2004; Cuijpers, 2015; Hollon et al., 2002; Hunot, Churchill, Teixeira, \& Silva de Lima, 2007; Schneider, Arch, \& WolitzkyTaylor, 2015; Vittengl, Clark, Dunn, \& Jarrett, 2007). While these therapies are effective in strictly controlled research settings, there is gathering evidence that such techniques are considerably less effective in "real-world" practice (see Goldfried et al., 2014). For example, Gibbons, Wiltsey Stirman, DeRubeis, Newman, and Beck (2013) reported that depressed individuals treated with cognitive therapy in a randomized controlled trial environment experienced a three-fold greater reduction in depressive symptoms, compared with similar people treated by the same therapists in a clinical setting. Such gaps between science and practice in psychotherapy have been variously attributed to differences in clinician training (Royal College of Psychiatrists, 2013), failure to adhere to standardized treatment protocols (Levita, Salas Duhne, Girling, \& Waller, 2016), or "therapist drift," wherein clinicians become less effective over time (Waller, 2009). Personality characteristics of the anxious or depressed individuals themselves have been identified as a challenge to the clinical application of evidence-based psychotherapies. Some of these challenging behaviors include resistance to doing homework (Westra, 2011), the presence of comorbid conditions and personality factors, and even the unwillingness to give up beliefs on the utility of worry, in the case of GAD (Szkodny, Newman, \& Goldfried, 2014). Therefore, the search for new and better treatment strategies for anxiety and depression is a high priority.

Affective psychological disorders have complex biological origins and are rarely the result of single insults, such as deficiencies in certain neurotransmitters, or focal lesions in specific brain locations. These disorders are instead characterized by abnormal electrical activity within networks of brain connections involving mood and behavior (Menon, 2011). Affective disorders can be influenced by abnormalities in intrinsic networks in the brain, such as the Default Mode Network (Broyd et al., 2009). Treatment protocols to harmonize the activity in such brain networks have the potential to mitigate the duration or severity of anxiety, depression, posttraumatic stress disorder, obsessive-compulsive disorder, or other affective disorders. Accumulating evidence suggests that neurofeedback therapy, which provides the clinician with the ability to modify and optimize aberrant brain wave activity in people with psychiatric conditions, can become a form of treatment in this field (Niv, 2013).

\section{Neurofeedback Training}

Neurofeedback is a form of biofeedback that provides live information about brain activity via electroencephalography (EEG) recordings from the scalp. Neurofeedback protocols are based on operant-conditioning paradigms and reward individuals when they increase or decrease the specific EEG component that is being "trained." These EEG components may include: brain waves (e.g., sensorimotor rhythm [SMR] training), ratios of brain waves (e.g., theta/beta ratio training), or connectivity between specific brain regions (e.g., coherence training). Without receiving any direct stimulation, individuals learn to optimize their brain activity to approach the target in the EEG component that is being rewarded. Repeated neurofeedback sessions reinforce or create new brain connections and pathways through the mechanism of neuroplasticity. These alterations correspond to positive changes in the individual's behavior and feelings (Niv, 2013).

Alpha-asymmetry (ALAY) training is one of the most common neurofeedback protocols for treatment of affective disorders (Baehr, Rosenfeld, \& Baehr, 2001), and it has been successfully applied to individuals with anxiety and/or depression. Choi et al. (2011) found positive results with the ALAY protocol in their pilot trial of 24 people with MDD. Participants in the active arm of this randomized, placebo-controlled pilot study who received 10 sessions of neurofeedback at F3 and F4 experienced significant improvement in their depression scores. In a small study with treatment of eight people who had an anxiety disorder, application of the ALAY protocol resulted in significant clinical benefits present 6 months after completion of treatment (Kerson, Sherman, \& Kozlowski, 2009).

Several other neurofeedback protocols have also been shown to be effective for individuals with anxiety and/or depression. Cheon, Koo, and Choi (2016) found that 8 weeks of neurofeedback therapy ( 2 or 3 times a week, in which first beta waves at F3 were increased for $30 \mathrm{~min}$ followed by $30 \mathrm{~min}$ of increasing the alpha/theta ratio at $\mathrm{Pz}$ ) significantly improved symptoms in people with a DSM-IV diagnosis of MDD. Among their 20 participants with MDD, $15 \%$ and $55 \%$ had remission of their condition 
at 4 weeks and 8 weeks, respectively. In a study of 24 people with multiple sclerosis who had significant depression and fatigue, Choobforoushzadeh, Neshat-Doost, Molavi, and Abedi (2015) provided half of participants with neurofeedback and the other half with "treatment as usual." Their neurofeedback protocol consisted of down-training theta and alpha waves, and up-training first beta and then SMR, at F3. They saw a statistically significant improvement only in the neurofeedback group, and these benefits were still present at 2-month follow-up evaluations. Another group of researchers (Sadjadi \& Hashemian., 2014) carried out a sham-controlled study to evaluate the benefits of 20 sessions of neurofeedback therapy in 24 children who had separation anxiety. Using a protocol that consisted of rewarding the alpha/theta ratio at F3, they found that children who received active neurofeedback treatment had less anxiety than the children who received the sham treatment. Walker and Lawson (2013) provided neurofeedback for 183 participants with MDD who were refractory to standard antidepressant medications. After six sessions of training in the right frontal-orbital area, rewarding a reduction of activity at $2-7 \mathrm{~Hz}$ and an increase of activity at $15-18 \mathrm{~Hz}$, remission or significant improvements were noted in $84 \%$ of participants. At follow-up 1 year after treatment, these improvements remained in effect for nearly all participants. Walker (2009) also reported positive results from a protocol to correct specific abnormalities seen on the quantitative electroencephalogram (qEEG) for 19 individuals who were diagnosed with posttraumatic stress disorder. A neurofeedback protocol based on qEEG was also shown to be successful for a group of 14 participants who had general anxiety disorder (Dreis et al., 2015) and in a group of 20 children who had anxiety due to the fact that they were removed from their homes by Child Protective Services (Huang-Storms, Bodenhamer-Davis, Davis, \& Dunn, 2006).

\section{Heart Rate Variability Training}

People with stress, anxiety, and depression have increased morbidity and mortality rates. This is likely due, in part, to increased activation of the sympathetic nervous system, which leads to increased incidence of cardiovascular disease. Increased cortisol released from the adrenal gland modulates the intrinsic neuronal pathways of the heart, which results in a higher pulse rate and reduced cardiac heart rate variability (HRV; Shaffer,
McCraty, \& Zerr, 2014). Activation of the sympathetic nervous system with stress and anxiety increases heart rate and is associated with low HRV. As would be expected, individuals with anxiety and difficulty in self-regulation of their emotions have high levels of sympathetic nervous system activity and low HRV (Williams et al., 2015). Recent studies have shown that vagal activation of the parasympathetic nervous system can slow down heart rate and enhance HRV. As such, a biofeedback modality that boosts the parasympathetic nervous system (e.g., deep slow abdominal breathing) can balance out the effects of sympathetic activation and increase HRV. In one study of 63 participants with coronary artery disease, training through deep abdominal breathing resulted in significant increase in HRV (Del Pozo, Gevirtz, Scher, \& Guarneri, 2004). HRV biofeedback has also been utilized as an intervention to treat disorders-such as depression, anxiety, PTSD, and hypertension-with quite promising, albeit preliminary, results (reviewed in Gevirtz, 2013).

\section{Combined Neurofeedback + Heart Rate} Variability Training

The combination of NFB protocols with HRV training (NFB+HRV) may help individuals optimize mood, cognitive performance, and the balance between sympathetic and parasympathetic functions. A small pilot study (Reid-Chung, Thompson, \& Thompson, 2015), found NFB+HRV training to be effective in reducing symptoms in participants with PostConcussive Syndrome. To our knowledge, no research study has examined the potential benefits of such combined therapy in individuals with anxiety and depression.

To determine whether the combination of neurofeedback training and heart rate variability training is a viable treatment strategy for individuals with symptoms of anxiety and depression, we administered NFB+HRV to clients with these symptoms. A parallel group of clients who did not meet the threshold for either anxiety or depression, but who had symptoms of other conditions (such as migraine or attention-deficit/hyperactivity disorder), also received this combined treatment. Before and after the NFB+HRV treatment protocol, EEG, blood pressure, breathing pattern, and psychological symptom measurements were taken for each client, and these pretreatment and posttreatment measurements were compared. 


\section{Methods}

Measurement of Psychological Symptoms

Achenbach System of Empirically Based Assessment. The Achenbach System of Empirically Based Assessment (ASEBA) symptom checklist was administered to measure the presence and severity of symptoms of anxiety and depression by questionnaire (Achenbach \& Rescorla, 2001). Adult clients completed the Adult Self-Report (ASR; Achenbach \& Rescorla, 2003), and parents completed the Child Behavioral Checklist (CBCL; Achenbach \& Rescorla, 2001) for children under age 18. The ASEBA provides $T$ scores for each behavioral scale. These $T$ scores are quantitative measures of the number and degree of symptoms reported, based on a gender- and age-normative database.

The ASEBA scale defines three possible conditions-Normal, Borderline, and Clinical-based on the degree of need for the individual to seek professional help (Figure 1).
Normal. Individuals have no need to seek professional help for anxiety or depression.

Borderline. Individuals have borderline need to seek professional help for anxiety or depression.

Clinical. Individuals need to seek professional help for anxiety or depression.

Deviant. Individuals with Borderline and Clinical designation can be combined as one Deviant group, to contrast them with individuals in the Normal group, who are deemed not to have any significant anxiety or depression symptoms. The ASEBA suggests using $T$ scores $<65$ to designate the Normal range vs. $T$ scores $\geq 65$ to designate the Deviant range (Achenbach \& Rescorla, 2001).

Because the term Deviant may cause offense when applied to individuals with psychological conditions, we instead refer to this group as Divergent herein.

\section{All Included Clients}

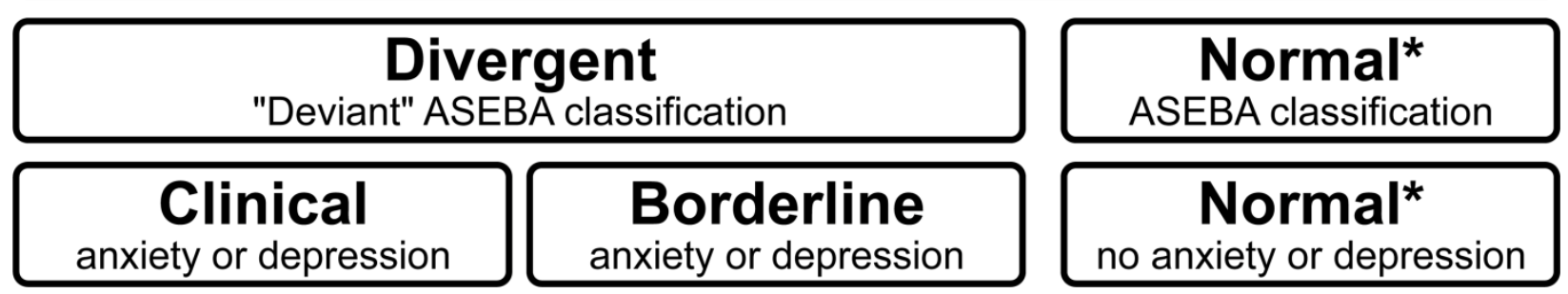

Figure 1. Diagram describing the ASEBA anxiety and depression classification system as it was utilized in this study. *These clients were Normal with regard to symptoms of anxiety and depression. However, all individuals in this study were clients of Neurocore, and likely experienced other symptoms such as migraine or ADHD.

\section{Clients}

The individuals in this study were child and adult clients of the Neurocore Brain Performance Center. Neurocore provides a combination of biofeedback and neurofeedback for individuals with a variety of symptoms, such as anxiety, depression, attentiondeficit/hyperactivity disorder (ADHD), autism, memory concerns, migraines, sleep disturbances, or stress. The research protocol for this study was approved by the New England Independent Review Board, which provided an IRB Privacy Board Waiver of Authorization to conduct a retrospective analysis of findings from clients who started a 30 -session $\mathrm{NFB}+\mathrm{HRV}$ treatment program on or after October
15,2015 , and completed the program by July 15 , 2016. All Personal Health Information Identifiers were removed from the dataset, which initially included a total of 378 clients. After exclusion criteria were applied, 334 clients remained in the current analysis. These criteria excluded eight individuals due to potential conflict of interest (employee or family member), three who were 60 years of age or older (due to age norms of the outcome measure), 10 who completed the program in less than 6 weeks or more than 6 months, and 14 extreme outliers who began the program with symptom rating scores higher than the $99^{\text {th }}$ percentile $(T$ score $>85)$ for symptoms of either 
anxiety or depression (see Assessment section above on the ASEBA).

This report consists of outcomes measured from clients treated at eight Neurocore centers in the Michigan cities: Bloomfield Hills, Grand Rapids, Grandville, Holland, Kalamazoo, Livonia, Okemos, and Sterling Heights. Of the 334 clients included, there were 123 females and 211 males. Clients were separated into two groups: adults and children.
Adults ranged in age from 18 to 59 , with an average age of 37.7 years $(S D=11.8)$. Children ranged in age from 6 to 17 , with an average age of 10.5 years $(S D=2.9)$. Table 1 depicts the demographic distribution of the 183 clients with ASEBA $T$ scores in the Divergent range for symptoms of anxiety, depression, or both at baseline (labeled Divergent) and 151 clients who did not meet the criteria for these conditions (labeled Normal).

\section{Table 1}

Baseline Demographics of Clients with Divergent ASEBA T scores for Anxiety, Depression, Both, or Neither at Baseline: Gender and Age

\begin{tabular}{|c|c|c|c|c|c|c|c|c|c|}
\hline & & \multicolumn{4}{|c|}{ Anxiety Only } & \multicolumn{4}{|c|}{ Depression Only } \\
\hline & & \multicolumn{2}{|c|}{ Adults } & \multicolumn{2}{|c|}{ Children } & \multicolumn{2}{|c|}{ Adults } & \multicolumn{2}{|c|}{ Children } \\
\hline & & $n$ & $M(S D)$ & $n$ & $M(S D)$ & $n$ & $M(S D)$ & $n$ & $M(S D)$ \\
\hline \multirow{3}{*}{ Age } & Female & 5 & $29.8(7)$ & 6 & $10.8(2)$ & 9 & $37.9(13)$ & 15 & $11.9(3)$ \\
\hline & Male & 7 & $38.4(12)$ & 17 & $9.9(1)$ & 8 & $28.0(9)$ & 35 & $11.8(3)$ \\
\hline & Total & 12 & $34.8(11)$ & 23 & $10.2(3)$ & 17 & $33.2(12)$ & 50 & 11.8 (3) \\
\hline
\end{tabular}

\begin{tabular}{|c|c|c|c|c|c|c|c|c|c|}
\hline & & \multicolumn{4}{|c|}{ Anxiety and Depression } & \multicolumn{4}{|c|}{ Normal } \\
\hline & & \multicolumn{2}{|c|}{ Adults } & \multicolumn{2}{|c|}{ Children } & \multicolumn{2}{|c|}{ Adults } & \multicolumn{2}{|c|}{ Children } \\
\hline & & $n$ & $M(S D)$ & $n$ & $M(S D)$ & $n$ & $M(S D)$ & $n$ & $M(S D)$ \\
\hline \multirow{3}{*}{ Age } & Female & 13 & $37.9(11)$ & 18 & $10.9(3.7)$ & 30 & $39.5(11.4)$ & 27 & $9.7(3.1)$ \\
\hline & Male & 6 & $30.8(11)$ & 44 & $10.2(2.6)$ & 25 & $42.4(11.9)$ & 69 & $10.1(2.8)$ \\
\hline & Total & 19 & $35.6(11)$ & 62 & $10.4(3)$ & 55 & $40.8(11.6)$ & 96 & $10.0(2.9)$ \\
\hline
\end{tabular}

Heart Rate Variability and Blood Pressure HRV was measured using a photoplethysmography sensor (Thought Technology Ltd., Montreal, Canada) attached to the client's index finger. Data were collected for 3 min using a sampling rate of $128 \mathrm{~Hz}$ with a ProComp2 or a ProComp5 amplifier, and BioGraph 5.1 software (Thought Technology Ltd., Montreal, Canada). A ProComp5 was used for all initial and final assessments; either a ProComp2 or a ProComp5 was used for individual sessions. Interbeat intervals were calculated from the raw signal using a low cutoff of $30 \mathrm{~ms}$ and a high cutoff of 2,000 ms, and a power spectrum was formed from these data using fast Fourier transform. HRV measures collected include the density (in $\mathrm{ms}^{2} / \mathrm{Hz}$ ) of the following frequency domains: very low frequency (VLF; $0.016-0.040 \mathrm{~Hz}$ ), low frequency (LF; $0.04-0.15 \mathrm{~Hz})$ and high frequency (HF; $0.15-$
$0.40 \mathrm{~Hz}$ ). Data were expressed as percentages of each frequency band, with respect to the overall range of frequencies collected $(0.016-0.500 \mathrm{~Hz})$. High-frequency HRV is largely driven by activity of the Parasympathetic Nervous System (The Task Force Report, 1996). Activation of the lowfrequency component of $\mathrm{HRV}$ is much more complex, with influences from both the Parasympathetic and the Sympathetic Nervous Systems (Billman, 2013). The VLF component is influenced by multiple homeostatic systems (including body temperature and circadian rhythms), and also by the heart itself (Shaffer et al., 2014). Respiration rate was measured by placing a straingauge belt (Thought Technology, Montreal, Canada) around the waist at the level of the umbilicus. Breaths per minute were calculated over the same 3-min intervals as HRV. 
Systolic blood pressure (SBP) and diastolic blood pressure (DBP) measurements were collected using a standard digital blood pressure monitor (A\&D Instruments, Abingdon, UK). Clients with either a systolic reading of at least $140 \mathrm{mmHg}$ or a diastolic reading of at least $90 \mathrm{mmHg}$ at baseline were defined as presenting with hypertension $(n=16$ within the Divergent group).

\section{Electroencephalography}

Electroencephalographic (EEG) assessment data were collected at $\mathrm{Cz}$ (a region within the sensorimotor cortex), positioned using the International 10/20 electrode system. Gold cup electrodes were placed in a monopolar montage, with the ground electrode on the right ear and the reference electrode on the left ear. The scalp site was cleaned with NuPrep skin prep gel, and the electrodes were adhered using Ten20 conductive paste (both from Weaver and Company, Aurora, CO). Data were collected for $90 \mathrm{~s}$ with a ProComp2 or a ProComp5 device, and BioGraph 5.1 software (Thought Technology Ltd., Montreal, Canada) using a sampling rate of $256 \mathrm{~Hz}$ after ensuring skin impedance levels were below $10 \mathrm{k} \Omega$. A ProComp5 device was used for all initial and final assessments, and either a ProComp2 or a ProComp5 device was used for individual sessions. Raw data were run through a Butterworth bandpass filter and average peak-to-peak amplitudes (in $\mu \mathrm{V}$ ) were calculated for the following frequency bands: theta $(4-8 \mathrm{~Hz})$, sensorimotor rhythm (SMR; $13-15 \mathrm{~Hz})$, low beta $(16-20 \mathrm{~Hz})$ and high beta $(23-35 \mathrm{~Hz})$. Two ratios were computed from these data: theta/beta ratio (theta/low beta) and high beta/SMR. These ratios were used to guide NFB training, as described below.

\section{Therapeutic Intervention}

Biofeedback and Neurofeedback. Clients included in the present report underwent 30 sessions of both neurofeedback and HRV training within a time period of 6 and 24 weeks. NFB+HRV training sessions were conducted by trained EEG technicians, under the supervision of licensed Masters of Social Work. Each feedback session began with 3 min of paced, slow breathing, with a goal of six to eight breaths per minute $(0.10-0.13$ $\mathrm{Hz}$ ), and HRV training. Breathing depth and rate were visualized on a 23- or 24-inch monitor along with fluctuations in heart rate interbeat interval, both fitted to a sinusoidal shape moving with time across the screen. Clients were coached in the use of diaphragmatic breathing and instructed to make the two sinusoidal curves overlap to achieve "coherence" between these measures, which up- trained the \%LF (low-frequency) band of HRV. Measurements collected at each session included average breaths per minute and \%LF band, used as a surrogate measure of HRV.

Following 3 min of HRV training, each client received a personalized NFB session based on their baseline theta/beta ratio and high beta/SMR ratio values at $\mathrm{Cz}$. The ProComp2 device and BioGraph 5.1 software were used to assess theta/beta ratio and high beta/SMR ratio in real time (frequencies and signal filtering detailed above), with feedback provided in the form of threshold-dependent presentation of a movie for a duration of $40 \mathrm{~min}$. Reward thresholds were set and enacted automatically in the form of the movie pausing using the following rules and feedback paradigms. Compared to the value measured at initial assessment, theta/beta ratio values were driven in the direction of the historical group average value of Neurocore's database of clients who had completed 30 sessions previously (theta/beta ratio $=2.35$ ). Therefore, clients with a baseline theta/beta ratio above 2.35 were trained to lower this ratio, and those with a theta/beta ratio below 2.35 were trained to raise it. High beta/SMR ratio values were consistently inhibited.

Two feedback mechanisms were provided simultaneously during each training session. One was a biofeedback mechanism for abdominal breathing rates, and the other was EEG feedback for brainwave activity. For respiratory biofeedback, if breathing had greater than $35 \%$ variation between breaths, then a negative stimulus of the video screen shrinking was provided. When clients were able to maintain variation of breathing under $35 \%$, the screen would remain in full screen mode, acting as a positive reinforcer. All clients were encouraged to maintain an average abdominal breathing rate between six to eight breaths a minute with less than $35 \%$ variation between breaths. When clients exceeded 8.75 breaths per minute, or their breathing pace fluctuations exceeded lagged thresholding criteria, the movie screen would shrink, using a transition time of $10 \mathrm{~s}$.

For EEG feedback, all treatment screens included positive feedback of the DVD playing for lowering high beta/SMR ratio (23-35/13-15) and maintaining theta/beta ratio $(4-8 / 16-20)$ within therapeutic ranges $(<3.0$ and $>1.7)$. A digital counter system within the BioGraph Infiniti software was employed to determine the percentage of reinforcement that was provided during training. An $80 \%( \pm 15 \%)$ success rate was used as a benchmark for training 
staff when choosing one of three available screens to begin the session (easy, average, difficult). Throughout the session, the software was configured to adjust between the three available screens seamlessly without notable interruption of DVD stimulus to maintain a reward rate of $80 \%$ $( \pm 15 \%)$. This ability to adjust treatment intensity screens while maintaining an $80 \%$ success rate was used to provide an achievable challenge for clients without overwhelming them.

Psychoeducation. All Neurocore clients received psychoeducation on a range of topics including sleep hygiene, diet, and exercise, in addition to learning coping skills like deep breathing. Roughly half of all clients in the present study $(n=176 / 334$ all clients; $n=96$ / 183 Divergent clients) met with a staff social worker for approximately 20 minutes before or after every session to review these topics. The remaining clients received similar educational input, but in a less formal manner. Because nonsignificant statistical regression models containing psychoeducation as a potentially confounding variable were not useful in predicting improvement in $T$ score, this variable was not included in the analyses that follow. The models are explained in the section below.

\section{Statistical Analysis}

All parametric statistical analyses were performed using SAS $^{\circledR}$ Enterprise Guide, Version 7.1. Calculations for confidence intervals for Cohen's effect sizes were performed using $R$ (a language and environment for statistical computing ( $R$ Core Team, 2016). Within $R$, the "irr" package was used to analyze the Stuart-Maxwell test of marginal homogeneity (Gamer, Lemon, Fellows, \& Singh, 2012). Other nonparametric statistical analyses, confidence intervals, and effect sizes were computed by hand. Statistical tables and formulae, from Applied Nonparametric Statistics (2nd ed.), were used to calculate test statistics, $p$-values, and confidence intervals (Daniel, 1990) for blood pressure analyses. A separate formula $r=\frac{Z}{\sqrt{N}}$ was used to compute the nonparametric effect sizes (Pallant, 2007). All $p$-values were assessed using an experiment-wise error rate of $\alpha=0.05$ adjusted for multiple testing and comparisons with Bonferroni correction. With 19 comparisons, the Bonferroni corrected significance level was $\alpha_{B}=0$. All tests performed were two-sided. Due to the fact that some clients in this retrospective study could be biologically related, the statistical assumption of independence may be questionable.
Potentially confounding variables, such as age, gender, center (i.e., the specific Neurocore branch attended by the client), presence or absence of formal psychoeducation, and test type (CBCL or ASR) were first investigated using multiple linear regression. For both anxiety and depression, the models containing age, gender, center, formal psychoeducation, and test type (CBCL or ASR) were not useful in predicting the magnitude of improvement in $T$ scores $(p=.129, .123$, respectively).

Mean $T$ score changes from pretreatment to posttreatment were assessed with paired t-tests. The normality assumption was satisfied given the large sample size; however, it was confirmed by assessing box plots and histograms.

The Minimal Clinically Important Difference (MCID) for the change in ASEBA scores is defined for two age ranges within each gender for each type of test; CBCL or ASR. The MCID is defined as the Standard Error of Measure (SE Meas). The SE Meas is calculated using statistics from ASEBA's age- and gender-normed population. The standard deviation is multiplied by the square root of the test retest reliability subtracted from one, SE Meas = $S D(\sqrt{1-\text { Reliability) }}$ (Achenbach \& Rescorla, 2003). Given that these values for MCIDs for anxiety and depression range from 1.65 to 2.55 (see Appendix, Supplemental Table 1), we conservatively defined an improvement of at least three points as the minimal clinically important difference.

Additionally, crosstabulation tables were produced to show the changes in $T$ scores for the three exhaustive categories per the ASEBA manual. The Stuart-Maxwell test for dependent proportions was used to test the statistical significance of the marginal homogeneity, or that row totals are equal to column totals, for all three classification levels from pretreatment to posttreatment (Everitt, 1992; Maxwell, 1970; Stuart, 1955). Assumptions for Stuart-Maxwell's were checked, with $\mathrm{K} \times \mathrm{K}$ mutually exclusive groups with pretreatment and posttreatment data and no categories with perfect agreement.

Due to the small number of clients in the anxiety and/or depression symptom Divergent groups who had hypertension at baseline (16/183) and the skewness of histogram of the differences from preto posttreatment, the nonparametric Wilcoxon Signed Rank test for paired differences was used to assess changes in blood pressure. 


\section{Results}

Anxiety and Depression Levels Pretreatment and Posttreatment

Assessment of all clients was performed with the ASEBA symptom checklist, both before and after the NFB+HRV training protocol. At baseline, 183 of the 334 clients had abnormal ASEBA scores for anxiety, depression, or both. These clients make up the Divergent group (Figure 1). ASEBA scores for the remaining 151 clients were within the Normal range for anxiety and depression symptoms (the Normal group). Among the Divergent group, 44\% $(n=81)$ presented with comorbid symptoms of both anxiety and depression. Of the 116 clients with Divergent ASEBA levels of anxiety symptoms (with or without comorbid depression symptoms), 35 exhibited symptoms of anxiety alone. Of the 148 clients with Divergent ASEBA levels of depression symptoms (with or without comorbid anxiety symptoms), 67 exhibited symptoms of depression alone.

Average change in $T$ score was investigated from pretreatment to posttreatment for clients with symptoms of anxiety only, depression only, or comorbid anxiety and depression at baseline. Paired t-tests were used to assess these changes. Those with pretreatment ASEBA $T$ scores in the Baseline or Clinical range for symptoms of anxiety only (without comorbid depression) experienced a significant decrease (improvement) in $T$ score of $M=$ 10.3 points after treatment, $S D=6.3,95 \% \mathrm{Cl}=[8.1$, 12.5], $t(34)=9.64, p<.001$. Similarly, those with pretreatment $T$ scores in the Baseline or Clinical range for symptoms of depression only (without comorbid anxiety) experienced a significant decrease in $T$ score of $M=8.8$ points after treatment, $S D=6.2,95 \% \mathrm{Cl}=[7.3,10.3], t(66)=$ 11.58, $p<.001$. Those with pretreatment $T$ scores in the Baseline or Clinical range for symptoms of both anxiety and depression experienced a significant decrease in anxiety $T$ score of $M=11.5$ points, $S D=8.4,95 \% \mathrm{Cl}=[9.6,13.4], t(80)=12.26$, $p<.001$; and a significant decrease in depression $T$ score of $M=10.4$ points, $S D=7.9,95 \% \mathrm{Cl}=[8.6$, 12.1], $t(80)=11.83, p<.001$.

With overlapping confidence intervals, there is not a statistically significant difference in the magnitude of change in $T$ score among clients with symptoms of anxiety only, depression only, or comorbid anxiety and depression at baseline. For this reason, further analyses address clients with symptoms of anxiety as well as clients with symptoms of depression, regardless of comorbidity. To address any effects of the client's age, gender, test type, formal psychoeducation condition, and Neurocore center on outcomes, a regression model was run containing all of these potential confounders (described in the Methods section). This model was not significant, with a Global $F$ test $p$-value of .129 for anxiety and .123 for depression. Separating the clients into subgroups based on these variables was, therefore, unnecessary. Additionally, client age and magnitude of improvement in $T$ score were not significantly correlated for anxiety $(r=-0.15, p$ $=.116)$ or depression $(r=-0.12, p=.135)$.

Table 2 shows the results of paired $t$-tests used to assess the average change in ASEBA $T$ score for those with Borderline or Clinical baseline symptoms of anxiety and depression. Those with pretreatment anxiety symptoms had a mean decrease in $T$ score of 11.1 points, $S D=7.8,95 \% \mathrm{Cl}=[9.7,12.6], t(115)$ $=15.28, \quad p<.001$; and those with pretreatment depression symptoms had a mean decrease in $T$ score of 9.7 points, $S D=7.2,95 \% \mathrm{Cl}=[8.5,10.8]$, $t(147)=16.32, p<.001$. The magnitude of these changes represents quite large effect sizes of $d_{z}=$ $1.42(95 \% \mathrm{Cl}=[1.16,1.68])$ for anxiety and $d_{\mathrm{z}}=1.34$ $(95 \% \mathrm{Cl}=[1.12,1.57])$ for depression. The mean $T$ scores before and after treatment for the two groups are displayed graphically (Figure 2). Table 2 also includes the percent of those who showed improvements of at least the MCID of three points. For anxiety, $82.8 \%$ improved by at least the MCID; for depression, $81.1 \%$ improved by at least the MCID. 
Table 2

ASEBA: Mean Changes for Clients in the Divergent Group at Baseline, Pretreatment to Posttreatment

\begin{tabular}{lccccccc}
\hline & & & \multicolumn{3}{c}{$M_{\mathrm{d}}\left(S D_{\mathrm{d}}\right)[95 \% \mathrm{Cl}]$} & \multicolumn{3}{c}{${\text { Improved } \geq \mathrm{MCID}^{\mathrm{a}}}$} \\
& $n$ & Pre & Post & Decrease & $d_{\mathrm{z}}$ & $p$ & $\%(n)$ \\
\hline Anxiety & 116 & $71.5(5.7)$ & $60.4(7.9)$ & $11.1(7.8)[9.7,12.6]$ & 1.42 & $<.001$ & $82.8 \%(96)$ \\
Depression & 148 & $70.9(4.8)$ & $61.2(8.0)$ & $9.7(7.2)[8.5,10.8]$ & 1.34 & $<.001$ & $81.1 \%(120)$ \\
\hline
\end{tabular}

Note. $M_{d}=$ Mean of differences; $S D_{d}=$ Standard deviation of differences; $d_{z}=$ Cohen's $d$ for effect size of paired differences

${ }^{a}$ An improvement in ASEBA $T$ score of at least the MCID of three.

\section{Average Pretreatment and Posttreatment $T$ Score Values for Clients in the Divergent Group at Baseline}

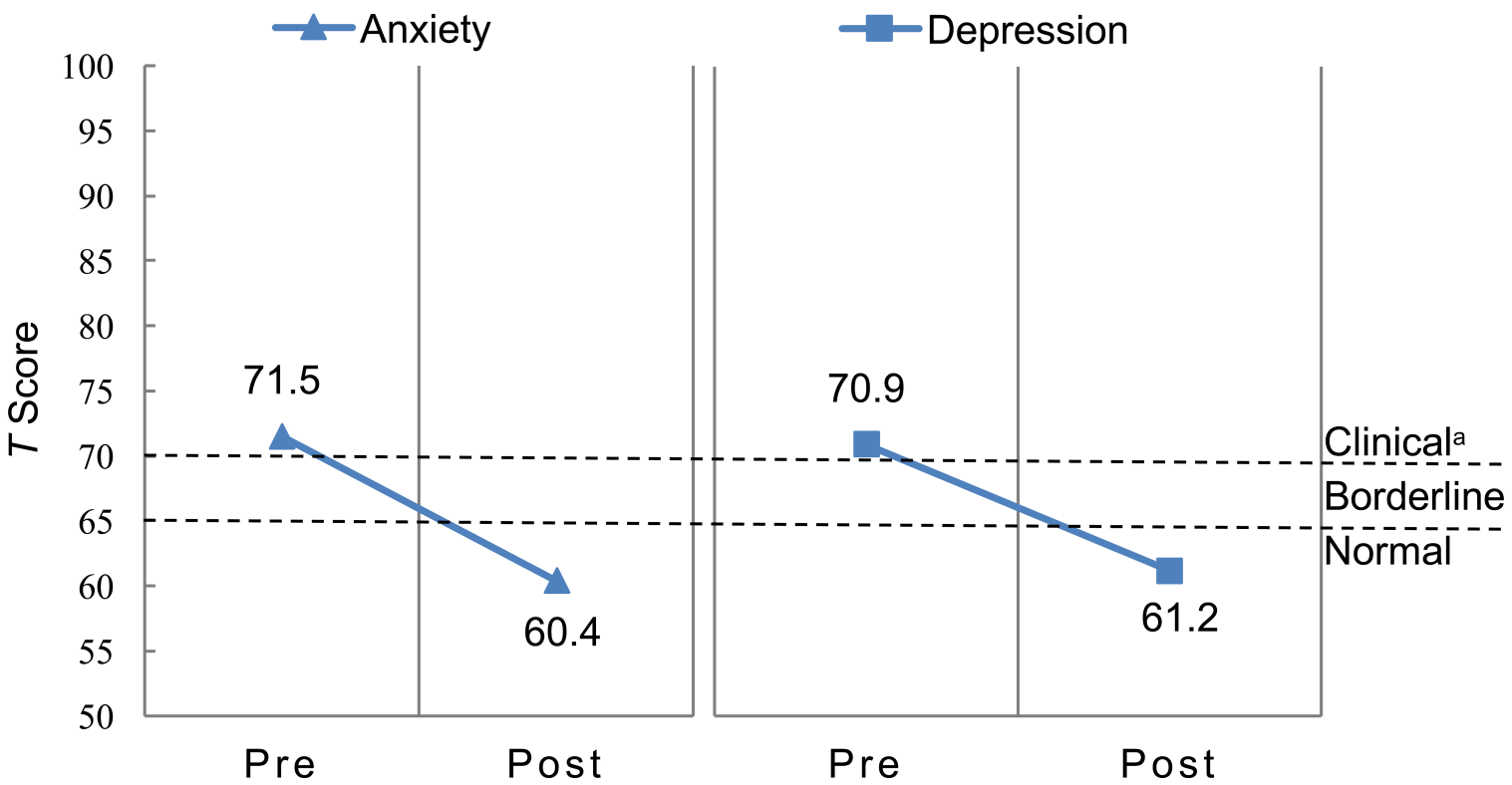

Figure 2. For clients in the Divergent Group at baseline: average ASEBA $T$ scores at pre-treatment and post-treatment are shown for anxiety and depression. For both anxiety and depression, these average values are in the Normal range after treatment, and the mean change is statistically significant. ${ }^{\mathrm{a}}$ Clinical Range $\geq 70$, Borderline Range 65-69, Normal Range $<65$, ASEBA's defined minimum possible $T$ score $=50$.

To assess whether the observed changes in ASEBA score were likely to be due to placebo, a subset of the most severe clients (those who presented at baseline with scores in the upper quartile of Divergent scores) were evaluated ( $T$ score $\geq 76$ ). Average $T$ scores were reduced by 15.11 points for those with anxiety symptoms, $n=28, S D=9.6,95 \%$ $\mathrm{Cl}=[11.40,18.81], t(27)=8.37, p<.001 ;$ and 13.29 points for those with depression symptoms, $n=24$, $S D=10.4,95 \% \mathrm{Cl}=[9.89,17.69], t(23)=6.25, p$ $<.001$, after treatment. For this anxiety symptom group, $89.3 \%$ of the upper quartile (25 out of 28 ) improved by at least the MCID of three, and $57.1 \%$ completely eliminated symptoms. The depression symptom group had similar results, with $79.2 \%$ of the upper quartile (19 out of 24) improving by at least the MCID of three, and $45.8 \%$ completely eliminating symptoms.

For both anxiety and depression, the Stuart-Maxwell test for marginal homogeneity was used to assess whether the proportion of clients with Clinical:Borderline:Normal ASEBA scores was different pretreatment compared to posttreatment. For each of the two tests run (one for anxiety and one for depression), all clients in the study were included. Each client was grouped according to whether they had Normal, Baseline, or Clinical 
ASEBA $T$ scores for the psychological disorder before treatment (pretreatment) and after treatment (posttreatment).

The proportion of clients with ASEBA $T$ scores in the Clinical:Borderline:Normal range significantly differed from pretreatment to posttreatment for both anxiety $\left(\chi^{2}=54.8, p<.001\right)$ and depression $\left(\chi^{2}=\right.$ $90.1, p<.001$; Table 3$)$. Specifically, the proportion of clients with ASEBA $T$ scores in the Clinical and Borderline range decreased from pretreatment to posttreatment, and the proportion of clients with ASEBA $T$ scores in the Normal range increased from pretreatment to posttreatment, for both anxiety and depression. For clients with symptoms of anxiety,
$60.3 \%$ of those with pretreatment Clinical status and $75.5 \%$ of those with pretreatment Borderline status experienced a posttreatment improvement in $T$ score sufficient to be considered Normal. Similarly, for clients with symptoms of depression, $50.6 \%$ of those with pretreatment Clinical status and $88.5 \%$ of those with pretreatment Borderline status experienced a posttreatment improvement in $T$ score sufficient to be considered Normal. Importantly, the majority of clients with pretreatment Clinical status for anxiety $(79.5 \%)$ and depression $(72.4 \%)$ were no longer in the Clinical range after treatment. These statistically significant results from the Stuart-Maxwell test are displayed graphically in Figure 3.

\section{Table 3}

Crosstabulation and Stuart-Maxwell Test of Marginal Homogeneity of ASEBA Classifications at Pretreatment by Posttreatment

\begin{tabular}{ccccc|ccc}
\hline \multirow{2}{*}{ Anxiety } & \multicolumn{7}{c}{$\begin{array}{c}\text { Post } \\
\text { Row Percent (Frequency) }\end{array}$} \\
\hline \multirow{3}{*}{ Pre } & Normal & Borderline & Clinical & Total & $\chi^{2}$ & $p$ \\
\cline { 3 - 8 } & Normal & $94.0 \%(205)$ & $5.1 \%(11)$ & $0.9 \%(2)$ & 218 & & \\
& Borderline & $75.5 \%(40)$ & $20.8 \%(11)$ & $3.8 \%(2)$ & 53 & & \\
& Clinical & $60.3 \%(38)$ & $19.1 \%(12)$ & $20.6 \%(13)$ & 63 & & \\
\cline { 2 - 9 } & Total & 283 & 34 & 17 & 334 & 54.8 & $<01$ \\
\hline
\end{tabular}

\begin{tabular}{|c|c|c|c|c|c|c|c|}
\hline & Depression & \multicolumn{3}{|c|}{ Post } & & & \\
\hline \multirow{5}{*}{ Pre } & & Normal & Borderline & Clinical & Total & $\chi^{2}$ & $p$ \\
\hline & Normal & $96.2 \%(179)$ & $3.8 \%(7)$ & $0.0 \%(0)$ & 186 & & \\
\hline & Borderline & $88.5 \%(54)$ & $8.2 \%(5)$ & $3.3 \%(2)$ & 61 & & \\
\hline & Clinical & $50.6 \%(44)$ & $21.8 \%(19)$ & $27.6 \%(24)$ & 87 & & \\
\hline & Total & 277 & 31 & 26 & 334 & 90.1 & $<.001$ \\
\hline
\end{tabular}


A Clients Borderline at Baseline

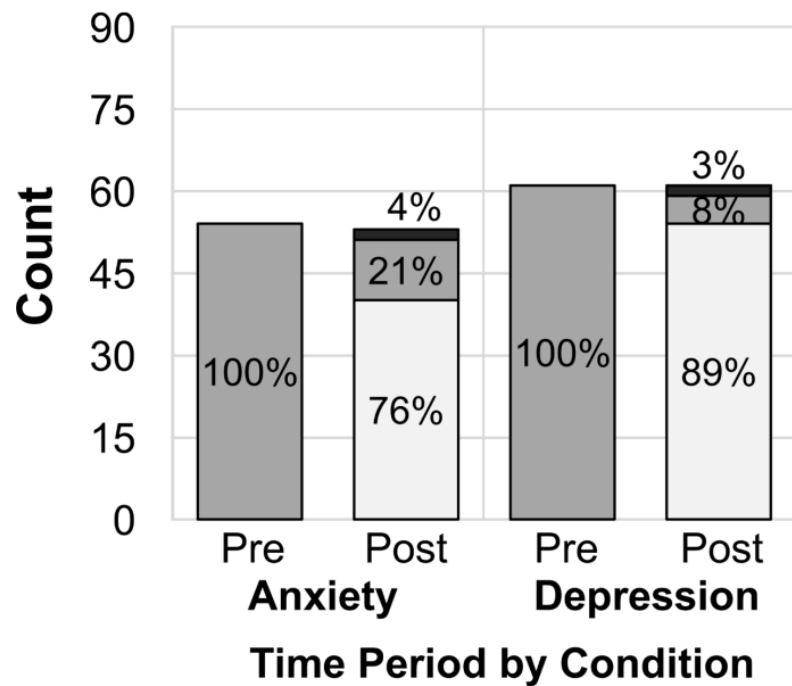

B Clients Clinical at Baseline

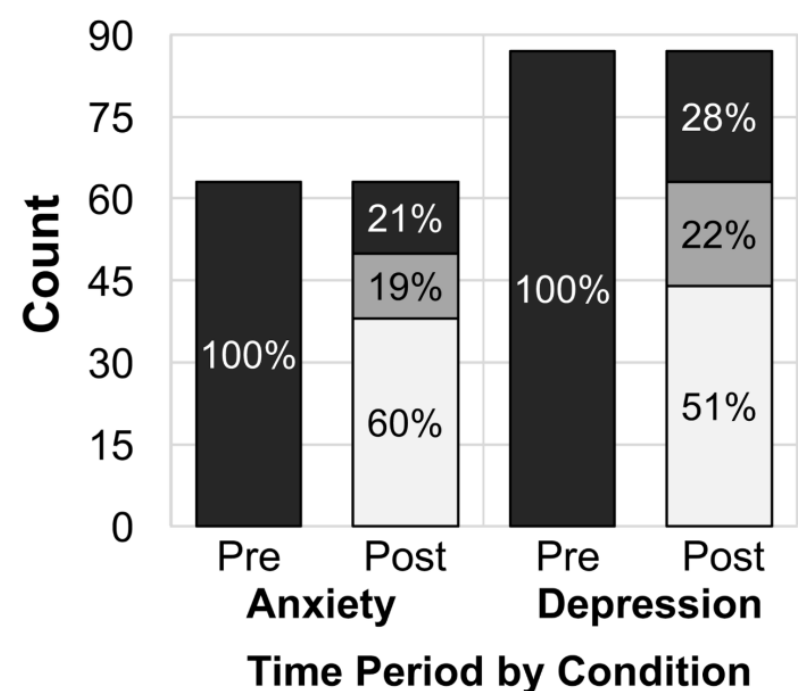

Time Period by Condition

\section{Normal \\ Borderline \\ Clinical}

Figure 3. The majority of clients with Divergent ASEBA scores at baseline were in the Normal group after NFB+HRV treatment. These results are statistically significant, based on the Stuart-Maxwell test displayed in Table 3 . (A) Clients in the pretreatment Borderline group for anxiety and depression are represented by the $100 \%$ Pre bars. After treatment (the Post bars), this group of clients was divided into Normal, Borderline, and Clinical groups, with the majority now in the Normal group. (B) The same is true for clients in the pretreatment Clinical group for both anxiety and depression.

\section{Heart Rate Variability and Blood Pressure}

HRV and Blood Pressure were recorded for a subset of clients (which includes 171 out of the 183 clients in the baseline Divergent group of clients with symptoms of anxiety and/or depression), due to missing data. HRV was evaluated using pretreatment to posttreatment changes in four measures: \%VLF (very low-frequency) band of the heart rate interbeat interval power spectrum, \%LF band, \%HF (high-frequency) band, and respiration rate (breaths per minute). All four measures were significantly different after treatment, with $p$-values less than .001 (Table 4). On average, \%VLF decreased by $6.1\left(d_{z}=-0.58\right)$, \%LF increased by $28.3\left(d_{z}=1.23\right)$, \%HF decreased by $19.3\left(d_{z}\right.$ $=-0.96)$, and respiration rate decreased by 6.4 breaths per minute $\left(d_{z}=-2.29\right)$.

\section{Table 4}

Mean Changes in Heart Rate Variability Measures from Pretreatment to Posttreatment for Clients in the Divergent Group at Baseline

\begin{tabular}{|c|c|c|c|c|c|c|}
\hline \multirow{3}{*}{$\begin{array}{l}n=171 \\
\% \text { VLF }\end{array}$} & \multicolumn{2}{|c|}{$M(S D)$} & $M_{\mathrm{d}}\left(S D_{\mathrm{d}}\right)$ & {$[95 \% \mathrm{Cl}]$} & \multirow[b]{2}{*}{$d_{\mathrm{z}}$} & \multirow[b]{2}{*}{$p$} \\
\hline & Pre & Post & \multicolumn{2}{|c|}{ Change } & & \\
\hline & $14.4(8.1)$ & $8.3(7.2)$ & $-6.1(10.5)$ & $-[4.5,7.6]$ & -0.58 & $<.001$ \\
\hline$\%$ LF & $34.2(12.7)$ & $62.6(21.8)$ & $28.3(23.1)$ & {$[24.8,31.8]$} & 1.23 & $<.001$ \\
\hline$\% \mathrm{HF}$ & $44.4(14.1)$ & $25.1(16.6)$ & $-19.3(20.1)$ & $-[16.2,22.3]$ & -0.96 & $<.001$ \\
\hline BPM & $14.0(2.0)$ & $7.6(2.3)$ & $-6.4(2.8)$ & $-[6.0,6.8]$ & -2.29 & $<.001$ \\
\hline
\end{tabular}

Note. $M_{\mathrm{d}}=$ Mean of differences; $S D_{\mathrm{d}}=$ Standard deviation of differences; $d_{\mathrm{z}}=$ Cohen's $\mathrm{d}$ for effect size of paired differences; $\mathrm{VLF}$ = very low-frequency; LF = low-frequency; HF = high-frequency; $B P M=$ breaths per minute. 
Changes in blood pressure were assessed for the baseline Divergent group of clients with symptoms of anxiety and/or depression who also presented with hypertension at baseline (those with either a systolic reading of at least $140 \mathrm{mmHg}$ or a diastolic reading of at least $90 \mathrm{mmHg} ; n=16$ ). These changes were assessed using the Wilcoxon Signed Rank test for matched pairs (Table 5). Significant improvement was found after treatment for both systolic and diastolic blood pressures, with $p$-values less than .001 . Systolic blood pressure improved with a median decrease of $14.0 \mathrm{mmHg}(Z=-3.15,94.94 \%$ $\mathrm{Cl}=[7.5,35.5], r=-0.56)$. Diastolic blood pressure had similar results, with a median decrease of 11.00 $\mathrm{mmHg}(Z=-3.1,94.94 \% \mathrm{Cl}=[.0,17.0], r=-0.59)$. Large effect sizes were found after NFB+HRV training by Cohen (1988) criteria (small $=0.1$, medium $=0.3$, large $=0.5$ )

\section{Table 5}

Median Changes in Blood Pressure from Pretreatment to Posttreatment for Clients in the Divergent Group with Hypertension at Baseline, Based on the Wilcoxon Test

\begin{tabular}{ccccccccc}
\hline \multirow{2}{*}{$n=16$} & \multicolumn{2}{c}{$M d n(I Q R)$} & & \multicolumn{2}{c}{$M d n_{d}\left(I Q R_{d}\right)$} & {$[94.94 \% \mathrm{Cl}]$} & & \\
\cline { 2 - 3 } & Pre & Post & & \multicolumn{2}{c}{ Change } & $d_{z}$ & $p$ \\
\hline SBP & $142.5(5.5)$ & $130.5(25.0)$ & & $-14.0(19.5)$ & $-[7.5,35.5]$ & -0.56 & $<.001$ \\
DBP & $87.5(12.0)$ & $74.5(10.5)$ & & $-11.0(14.5)$ & $-[5.0,17.0]$ & -0.59 & $<.001$ \\
\hline
\end{tabular}

Note. SBP = systolic blood pressure; DBP = diastolic blood pressure; $M d n=$ median; $I Q R=$ Interquartile Range; $M d n_{\mathrm{d}}=$ Median of differences; $I Q R_{\mathrm{d}}=$ Interquartile Range of differences.

\section{EEG}

For clients in the Divergent groups at baseline, changes in two ratios of interest (high beta/SMR and theta/beta) from pretreatment to posttreatment EEGs were assessed (Table 6). Baseline Divergent group clients experienced an average decrease in high beta/SMR ratio of $0.13, S D=0.26,95 \% \mathrm{Cl}=[0.09$, $0.17], d_{z}=0.500, t(182)=6.79, p<.001$. Because the NFB protocol drove the theta/beta ratio toward Neurocore's historic client group average value of 2.35 (see Methods), clients were split into two groups in order to assess whether the theta/beta ratio moved in the "expected" direction. One group included clients with a baseline theta/beta ratio above 2.35, and one group included those below 2.35. Clients with baseline Divergent scores for depression and/or anxiety symptoms, and who started out with a theta/beta ratio below 2.35 , had an average increase of 0.19 after treatment, $S D=0.37$, $95 \% \mathrm{Cl}=[0.08,0.29], d_{z}=-0.513, t(51)=3.63, p$ $=.001$. Clients with baseline Divergent scores in depression and/or anxiety symptoms, and who started out with a theta/beta ratio above 2.35 did not experience a statistically significant change from baseline, $t(131)=-1.45, p=.149$.

\section{Table 6}

Mean Changes in EEG Ratios for Clients with Divergent Scores at Baseline in Anxiety and/or Depression

\begin{tabular}{|c|c|c|c|c|c|c|c|}
\hline & \multirow[b]{2}{*}{$n$} & \multicolumn{2}{|c|}{$M(S D)$} & $M_{\mathrm{d}}(S D)$ & [95 \% Cl] & & \\
\hline & & Pre & Post & \multicolumn{2}{|c|}{ Change } & $d_{z}$ & $p$ \\
\hline$\frac{H B}{S M R}$ & 183 & $1.43(0.36)$ & $1.30(0.31)$ & $-0.13(0.26)$ & {$[-0.09,-0.17]$} & 0.500 & $<.001$ \\
\hline$\frac{\text { Theta }}{\text { Beta }}<2.35$ & 52 & $1.88(0.31)$ & $2.07(0.46)$ & $0.19(0.37)$ & {$[0.29,0.08]$} & -0.513 & $<.001$ \\
\hline$\frac{\text { Theta }}{\text { Beta }}>2.35$ & 131 & $3.15(0.57)$ & $3.22(0.74)$ & $0.07(0.53)$ & {$[-0.16,0.02]$} & 0.132 & .149 \\
\hline
\end{tabular}

Note. $\mathrm{HB} / \mathrm{SMR}=$ high beta/sensorimotor rhythm. 


\section{Discussion}

In the present retrospective study, we found that clients who suffered from symptoms of anxiety and/or depression experienced substantial improvement in symptoms after 30 sessions of $\mathrm{NFB}+\mathrm{HRV}$ training. The majority of clients with pretreatment symptoms of anxiety $(82.8 \%)$ or symptoms of depression (81.1\%) experienced ASEBA $T$ score improvements of clinical importance after treatment (by at least the MCID of three; see Methods). Most importantly, the majority of clients with baseline Divergent scores for anxiety or depression symptoms were in the Normal group after NFB+HRV treatment. Even for the clients for whom anxiety and depression symptoms were the most severe (the upper quartile of pretreatment $T$ score), $57.1 \%$ of those with symptoms of anxiety and $45.8 \%$ of those with symptoms of depression were in the Normal group after treatment.

Neurofeedback can enhance the function of neuronal networks associated with mood and behavior (Simkin, Thatcher, \& Lubar, 2014), and lead to alterations in brain structure that are observable via magnetic resonance imaging (MRI; Ghaziri et al., 2013). Ghaziri et al. performed MRI on participants before and after a course of treatment with NFB, which indicated that parts of the frontal lobe and association cortical areas increased in size. Although the majority of prior work in anxiety and depression neurofeedback involves modification of the alpha frequency band, the present study is unique in that we trained two ratios of frequencies that do not involve alpha: high beta/SMR ratio and theta/beta ratio.

Our protocol inhibited the high beta/SMR ratio, and comparison of pre- and postprogram EEG found that clients with symptoms of anxiety and/or depression had an average decrease of 0.13 in high beta/SMR ratio $\left(p<.001, d_{z}=0.500\right)$. This was the expected result, based on our specific NFB protocol. Because our protocol inhibited a ratio metric (high beta/SMR) rather than individual frequency bands, we cannot say whether the decrease in this ratio after $\mathrm{NFB}+\mathrm{HRV}$ treatment was due to an increase in SMR, a decrease in high beta, or both. However, any of these changes would be predicted to improve mood. There is a large body of literature to support the benefits of increasing SMR at $\mathrm{Cz}$ (Sterman, 1996), while high beta is a frequency band associated with rumination, obsessional thoughts, and anxiety (Thompson \& Thompson, 2006). Lowering high beta has been shown to reduce symptoms of anger (Walker, 2013) and is often used as an inhibit frequency in traditional NFB training paradigms (see Walker [2009] as an example).

Our NFB protocol was also designed to drive the theta/beta ratio toward Neurocore's historic client group average (2.35). For pretreatment Divergent group clients who had a theta/beta ratio below this value at baseline, there was a statistically significant increase in theta/beta ratio $(p<.001)$, which was the expected result based on the protocol. For those with a theta/beta ratio greater than the historical group average at baseline, there was no significant change in theta/beta ratio after treatment. Based on our NFB protocol, we would have expected this ratio to go down. Therefore, the theta/beta ratio moved in the expected direction for some clients but not others, and we succeeded only in raising theta/beta. Because our protocol targeted a ratio metric (theta/beta), we cannot say whether this increase was due to an increase in theta, a decrease in beta, or both. Although an elevated theta/beta ratio has long been associated with ADHD (Arns, Conners, \& Kraemer, 2013), this may not be the case for anxiety and depression. A low theta/beta ratio measured at O1 over the left occipital has been associated with poor quality of sleep and a feeling of exhaustion (Swingle, 2015). Although this has not yet been formally tested, in our clinical experience we have found that this association may also hold true for low theta/beta ratio measured at $\mathrm{Cz}$ (TGR, unpublished observations). If so, raising the theta/beta ratio in this subset of clients may also improve symptoms of anxiety and depression by relieving exhaustion. That being said, Walker and Lawson (2013) have reported improvements in depressive symptoms following an NFB protocol that lowered theta and raised beta $(15-18 \mathrm{~Hz})$ at FPO2.

Clients in this study with baseline anxiety or depression also experienced changes in breathing rate and in relative HRV frequency spectrum that were consistent with our NFB+HRV protocol. We trained both breathing rate and HRV to the range of six to eight cycles per minute $(0.10-0.13 \mathrm{~Hz})$. This corresponds to a breathing rate of six to eight breaths per minute and HRV in the low-frequency band (see Methods). Indeed, after treatment the average resting breathing rate was 7.6 breaths per minute (which was significantly decreased from the pretreatment value of 14.0), and the \%LF band of the heart rate interbeat interval power spectrum was significantly increased. Importantly, there were also significant decreases in both systolic and diastolic blood pressure for pretreatment Divergent group clients who had hypertension at baseline. 
The inclusion of HRV training before every NFB session might have contributed to the decreased psychological symptom severity and the other physiological changes observed in this study. HRV training is thought to work by enhancing the parasympathetic influences on the heart, producing significant benefits on cardiac function and HRV, although several theories about specific mechanism currently exist (Reviewed in Shaffer et al., 2014). In the present study, we found a significant increase in the \%LF band of the heart rate interbeat interval power spectrum. Power increases within this band have been associated with strengthening the baroreflex system, which mediates reciprocal changes between blood pressure and heart rate (Lehrer, 2013). In support of this, we observed a significant decrease in blood pressure for clients who had elevated blood pressure levels at baseline. \%LF range power increases could also be due to a shift in the frequency of Respiratory Sinus Arrhythmia (RSA), which usually corresponds to breathing frequency (Yasuma \& Hayano, 2004).

RSA increases blood flow to oxygen-rich lung alveoli by raising heart rate during intake of breath and reduces blood flow to oxygen-poor alveoli by lowering heart rate during exhalation (Lehrer \& Gevirtz, 2014; Vaschillo, Lehrer, Rishe, \& Konstantinov, 2002). Individuals trained with biofeedback techniques to maximize the amplitude of RSA usually learn to achieve this by breathing at a rate of approximately six breaths per minute (Lehrer, Vaschillo, \& Vaschillo, 2000), and intentional paced breathing at this frequency can produce very high-amplitude HRV (Vaschillo et al.,
2002). One study in which HRV parameters were measured while participants breathed at specified rates found that total HRV amplitude peaked at four breaths per minute, as did low-frequency HRV amplitude (Song \& Lehrer, 2003). This is also within the breathing frequency range utilized by Zen monks for the practice of "tanden breathing," during which their HRV increases in the low-frequency band, and decreases in the high-frequency band (Lehrer, Sasaki, \& Saito, 1999). The NFB+HRV protocol utilized in the current study combined biofeedback to encourage slow, deep breathing (at 6-8 breaths per minute) with HRV biofeedback to up-train the \%LF band. Because RSA strength is postulated to represent an "index" of total cardiac vagal tone (Porges, 2007), it may be that the increase in \%LF band that we observe is associated with enhanced activity of the parasympathetic nervous system. The current study was not designed to look at absolute changes within the HRV power spectrum, however, so no specific claims for a mechanism can be made.

The effects of HRV training are not thought to be solely on heart function. A recent study showed that healthy individuals who have an optimal highfrequency HRV often possess thicker cortex in the right anterior cingulate cortex (Winkelmann et al., 2016). Combining the NFB and HRV forms of biofeedback, which impact the physiology of both the central and autonomic nervous systems, may be the main reason we have seen such robust clinical benefits in our clients. Based on the results of the current study, as well as others in the field, we propose a model for how NFB and HRV interact to enhance mood and energy (Figure 5). 


\section{Neurofeedback Training}

- Optimizes brain wave patterns

- Enhances neuronal connections and networks, leads to relaxation

- Improves activity in Parasympathetic Nervous System, slows down heart rate and breathing rate

- Lowers blood pressure

\section{(n)}

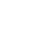

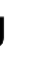

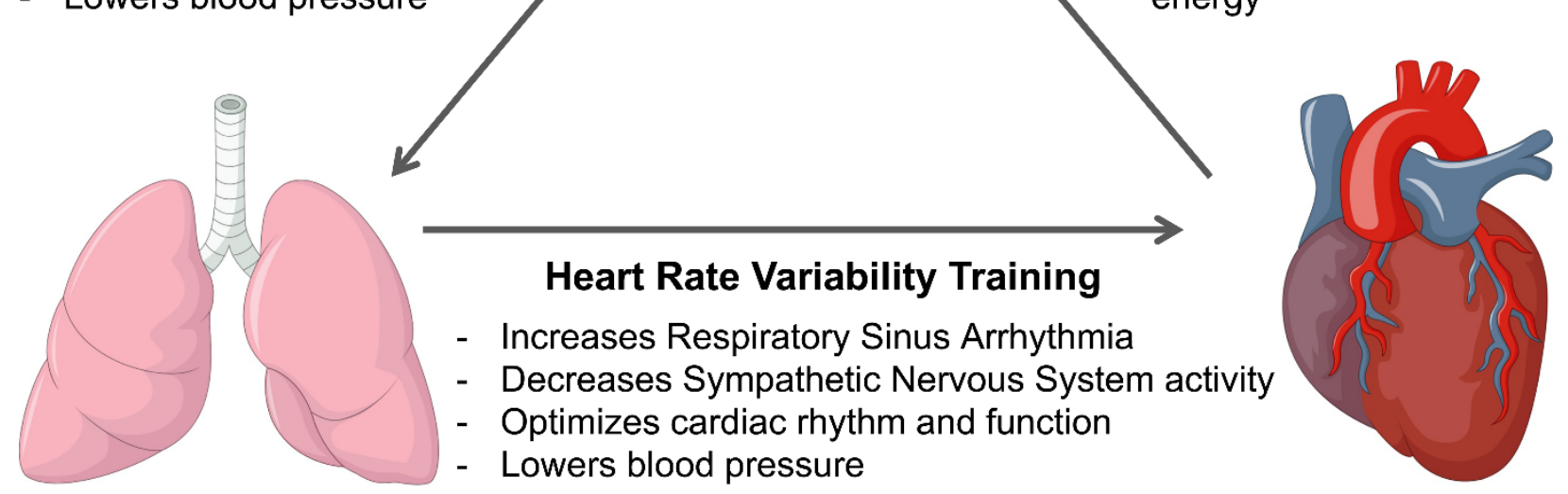

Figure 5. Model describing how concurrent neurofeedback and heart rate variability biofeedback training may interact to improve the function of both the nervous and cardiovascular systems.

Some studies (Khan \& Brown, 2015) suggest that individuals with mild to moderate depression are prone to experience clinically significant improvement in symptoms due to placebo effects. Indeed, low symptom severity is among the best predictors of a large placebo effect for many different psychological conditions (Weimer, Colloca, \& Enck, 2015). However, in this study, even those with severe symptoms gained clinically meaningful benefits from NFB+HRV. The presence of statistically significant changes in objective physiological parameters, such as EEG, HRV oscillations, and blood pressure, further suggests that improvements in ASEBA score (which are based on clients' subjective impression of their symptoms) might not be due to placebo effect. Finally, the large magnitude of effect sizes for both clients with anxiety and depression symptoms suggests the benefits of this treatment protocol.

A main strength of this study was combining NFB with HRV training for treating a large number of both adults and children $(n=183)$ with anxiety and/or depression. Because significant changes were found after training for both HRV variables as well as EEG ratios, it is likely that both of these interventions contributed to the significant decrease in symptom presence and severity. Another strength is the fact that data were collected from a geographically diverse cohort (in one of eight different Michigan cities). Interestingly, we found no difference in ASEBA or physiological outcome between clients of different age, gender, test type, or Neurocore center, which may indicate that our NFB+HRV protocol could be effective for clients from many different demographics.

Limitations of this study include its retrospective design and the lack of a sham control group. Further, a limited EEG, and not a full-cap 19electrode quantitative EEG, was utilized to analyze brain wave activity at baseline and follow-up visits. Rather than training individual rhythms, our NFB protocol trained two ratio metrics; this means that for a given change in the trained ratio, we did not distinguish whether this was accomplished by a change in the numerator rhythm, an opposing change in the denominator rhythm, or both. 
Measurement of psychological symptom presence and severity in this study was based on the ASEBA, which does not finely distinguish between subtypes of anxiety or depression (e.g., posttraumatic stress disorder or obsessive compulsive disorder). Any differential effects of the NFB+HRV protocol on various subtypes of anxiety and depression could therefore have been missed. For the HRV portion of the study, our study design enabled us to consider only relative changes within the HRV power spectrum (rather than absolute changes). Clients were also not reexamined after the conclusion of the program to determine whether the post-NFB+HRV changes were long-lasting. Finally, due to our study design, we were unable to distinguish between the potential benefits of NFB+HRV treatment versus either NFB or HRV treatment alone. Although some factors, such as our large sample size, robust effect size, use of standard diagnostic DSMV criteria and ASEBA scores, and presence of physiological biomarkers mitigate the negative impact of these limitations, a prospective, blinded study with appropriate sham control group, more stringent inclusion criteria, and long-term follow-up is needed to determine whether NFB+HRV can indeed produce robust and long-lasting results.

\section{Conclusion}

This report is the first to show that NFB+HRV training may have a robust effect on improving symptoms of anxiety and depression. NFB+HRV training may also improve physiological functions within the autonomic nervous system and cardiovascular system, including blood pressure and heart rate variability. Further prospective placebocontrolled longitudinal clinical trials are warranted.

\section{Author Note}

SAS and all other SAS Institute Inc. product or service names are registered trademarks or trademarks of SAS Institute Inc. in the USA and other countries. $₫$ indicates USA registration.

\section{Acknowledgements}

The authors thank Yahya Alshamy and Parnia Ghazanfari for their contributions to this study.

\section{Financial Interest}

All of the authors are employees of, or consultants with, the companies Neurocore or Neurocore Pro, Grand Rapids, Michigan, USA.

\section{References}

Abejuela, H. R., \& Osser, D. N. (2016). The Psychopharmacology Algorithm Project at the Harvard South Shore Program: An Algorithm for Generalized Anxiety Disorder. Harvard Review of Psychiatry, 24(4), 243-256. http://dx.doi.org/10.1097 /HRP.0000000000000098

Achenbach, T. M., \& Rescorla, L. A. (2001). Manual for the ASEBA School-Age Forms and Profiles. Burlington, VT: University of Vermont, Research Center for Children, Youth, \& Families.

Achenbach, T. M., \& Rescorla, L. A. (2003). Manual for the ASEBA Adult Forms and Profiles. Burlington, VT: University of Vermont, Research Center for Children, Youth, \& Families.

Andrews, G., Issakidis, C., Sanderson, K., Corry, J., \& Lapsley, H. (2004). Utilising survey data to inform public policy: Comparison of the cost-effectiveness of treatment of ten mental disorders. The British Journal of Psychiatry, 184(6), 526-533. http://dx.doi.org/10.1192/bjp.184.6.526

Arns, M., Conners, C. K., \& Kraemer, H. C. (2013). A Decade of EEG Theta/Beta Ratio Research in ADHD: A Meta-Analysis. Journal of Attention Disorders, 17(5), 374-383. http://dx.doi.org/10.1177/1087054712460087

Asselmann, E., \& Beesdo-Baum, K. (2015). Predictors of the Course of Anxiety Disorders in Adolescents and Young Adults. Current Psychiatry Reports, 17, 7 . http://dx.doi.org/10.1007/s11920-014-0543-z

Baehr, E., Rosenfeld, J. P., \& Baehr, R. (2001). Clinical Use of an Alpha Asymmetry Neurofeedback Protocol in the Treatment of Mood Disorders. Journal of Neurotherapy, 4(4), 11-18. http://dx.doi.org/10.1300/J184v04n04_03

Billman, G. E. (2013). The LF/HF ratio does not accurately measure cardiac sympatho-vagal balance. Frontiers in Physiology, 4, 26. http://dx.doi.org/10.3389/fphys.2013.00026

Broyd, S. J., Demanuele, C., Debener, S., Helps, S. K., James, C. J., \& Sonuga-Barke, E. J. S. (2009). Default-mode brain dysfunction in mental disorders: A systematic review. Neuroscience \& Biobehavioral Reviews, 33(3), 279-296. http://dx.doi.org/10.1016/j.neubiorev.2008.09.002

Carvalho, A. F., Sharma, M. S., Brunoni, A. R., Vieta, E., \& Fava, G. A. (2016). The Safety, Tolerability and Risks Associated with the Use of Newer Generation Antidepressant Drugs: A Critical Review of the Literature. Psychotherapy and Psychosomatics, 85(5), 270-288. http://dx.doi.org/10.1159 1000447034

Cheon, E.-J., Koo, B.-H., \& Choi, J.-H. (2016). The Efficacy of Neurofeedback in Patients with Major Depressive Disorder: An Open Labeled Prospective Study. Applied Psychophysiology and Biofeedback, 41(1), 103-110. http://dx.doi.org/10.1007/s10484-015-9315-8

Choi, S. W., Chi, S. E., Chung, S. Y., Kim, J. W., Ahn, C. Y., \& $\mathrm{Kim}, \mathrm{H}$. T. (2011). Is alpha wave neurofeedback effective with randomized clinical trials in depression? A pilot study. Neuropsychobiology, 63(1), 43-51. http://dx.doi.org/10.1159 1000322290

Choobforoushzadeh, A., Neshat-Doost, H. T., Molavi, H., \& Abedi, M. R. (2015). Effect of neurofeedback training on depression and fatigue in patients with multiple sclerosis. Applied Psychophysiology and Biofeedback, 40(1), 1-8. http://dx.doi.org/10.1007/s10484-014-9267-4

Cohen, J. (1988). Statistical power analysis for the behavioral sciences (2nd ed.). Hillsdale, $\mathrm{NJ}$ : Lawrence Erlbaum Associates.

Cuijpers, P. (2015). Psychotherapies for adult depression. Current Opinion in Psychiatry, 28(1), 24-29. http://dx.doi.org/10.1097 YYCO.0000000000000121

Daniel, W. W. (1990). Applied Nonparametric Statistics (2nd ed.). Boston, MA: Brooks/Cole Cengage Learning.

Del Pozo, J. M., Gevirtz, R. N., Scher, B., \& Guarneri, E. (2004). Biofeedback treatment increases heart rate variability in 
patients with known coronary artery disease. American Heart Journal, 147(3), 545. http://dx.doi.org/10.1016 /j.ahj.2003.08.013

Dreis, S. M., Gouger, A. M., Perez, E. G., Russo, G. M., Fitzsimmons, M. A., \& Jones, M. S. (2015). Using Neurofeedback to Lower Anxiety Symptoms Using Individualized qEEG Protocols: A Pilot Study. NeuroRegulation, 2(3), 137-148. http://dx.doi.org /10.15540/nr.2.3.137

Everitt, B. S. (1992). The Analysis of Contingency Tables (2nd ed.). London, UK: Chapman \& Hall.

Gamer, M., Lemon, J., Fellows, I., \& Singh, P. (2012). irr: Various Coefficients of Interrater Reliability and Agreement (Version 0.84). Retrieved from https://cran.r-project.org/package=irr

Gevirtz, R. (2013). The Promise of Heart Rate Variability Biofeedback: Evidence-Based Applications. Biofeedback, 41(3), 110-120. http://dx.doi.org/10.5298/1081-5937-41.3.01

Ghaziri, J., Tucholka, A., Larue, V., Blanchette-Sylvestre, M., Reyburn, G., Gilbert, G., ... Beauregard, M. (2013). Neurofeedback training induces changes in white and gray matter. Clinical EEG and Neuroscience, 44(4), 265-272. http://dx.doi.org/10.1177/1550059413476031

Gibbons, C. R., Wiltsey Stirman, S., DeRubeis, R. J., Newman, C. F., \& Beck, A. T. (2013). Research Setting Versus Clinic Setting: Which Produces Better Outcomes in Cognitive Therapy for Depression? Cognitive Therapy and Research, 37(3), 605-612. http://dx.doi.org/10.1007/s10608-012-9499-7

Goldfried, M. R., Newman, M. G., Castonguay, L. G., Fuertes, J. N., Magnavita, J. J., Sobell, L., \& Wolf, A. W. (2014). On the Dissemination of Clinical Experiences in Using Empirically Supported Treatments. Behavior Therapy, 45(1), 3-6. http://dx.doi.org/10.1016/j.beth.2013.09.007

Hollon, S. D., Muñoz, R. F., Barlow, D. H., Beardslee, W. R., Bell, C. C., Bernal, G., ... Sommers, D. (2002). Psychosocial intervention development for the prevention and treatment of depression: Promoting innovation and increasing access. Biological Psychiatry, 52(6), 610-630. http://dx.doi.org /10.1016/S0006-3223(02)01384-7

Huang-Storms, L., Bodenhamer-Davis, E., Davis, R., \& Dunn, J. (2006). QEEG-Guided Neurofeedback for Children with Histories of Abuse and Neglect: Neurodevelopmental Rationale and Pilot Study. Journal of Neurotherapy, 10(4), 316. http://dx.doi.org/10.1300/J184v10n04_02

Hunot, V., Churchill, R., Teixeira, V., \& Silva de Lima, M. (2007). Psychological therapies for generalised anxiety disorder. Cochrane Database of Systematic Reviews, 1, CD001848. http://dx.doi.org/10.1002/14651858.CD001848.pub4

Kerson, C., Sherman, R. A., \& Kozlowski, G. P. (2009). Alpha Suppression and Symmetry Training for Generalized Anxiety Symptoms. Journal of Neurotherapy, 13(3), 146-155. http://dx.doi.org/10.1080/10874200903107405

Kessler, R. C., Angermeyer, M., Anthony, J. C., De Graaf, R., Demyttenaere, K., Gasquet, I., ... Üstün, T. B. (2007). Lifetime prevalence and age-of-onset distributions of mental disorders in the World Health Organization's World Mental Health Survey Initiative. World Psychiatry, 6(3), 168-176.

Khan, A., \& Brown, W. A. (2015). Antidepressants versus placebo in major depression: An overview. World Psychiatry, 14(3), 294-300. http://dx.doi.org/10.1002/wps.20241

Kirsch, I., Deacon, B. J., Huedo-Medina, T. B., Scoboria, A. Moore, T. J., \& Johnson, B. T. (2008). Initial Severity and Antidepressant Benefits: A Meta-Analysis of Data Submitted to the Food and Drug Administration. PLoS Medicine, 5(2), e45. http://dx.doi.org/10.1371/journal.pmed.0050045

Lehrer, P. M. (2013). How Does Heart Rate Variability Biofeedback Work? Resonance, the Baroreflex, and Other Mechanisms. Biofeedback, 41(1), 26-31. http://dx.doi.org /10.5298/1081-5937-41.1.02

Lehrer, P. M., \& Gevirtz, R. (2014). Heart rate variability biofeedback: How and why does it work? Frontiers in
Psychology, 5, 756. http://dx.doi.org/10.3389 /fpsyg.2014.00756

Lehrer, P. M., Sasaki, Y., \& Saito, Y. (1999). Zazen and cardiac variability. Psychosomatic Medicine, 61(6), 812-821. http://dx.doi.org/10.1097/00006842-199911000-00014

Lehrer, P. M., Vaschillo, E., \& Vaschillo, B. (2000). Resonant frequency biofeedback training to increase cardiac variability: rationale and manual for training. Applied Psychophysiology and Biofeedback, 25(3)P, 177-191. http://dx.doi.org/10.1023 la:1009554825745

Levita, L., Salas Duhne, P. G., Girling, C., \& Waller, G. (2016). Facets of clinicians' anxiety and the delivery of cognitive behavioral therapy. Behaviour Research and Therapy, 77, 157-161. http://dx.doi.org/10.1016/j.brat.2015.12.015

Maxwell, A. E. (1970). Comparing the classification of subjects by two independent judges. The British Journal of Psychiatry, 116(535), 651-655. http://dx.doi.org/doi:10.1192 /bjp.116.535.651

Menon, V. (2011). Large-scale brain networks and psychopathology: A unifying triple network model. Trends in Cognitive Sciences, 15(10), 483-506. http://dx.doi.org /10.1016/j.tics.2011.08.003

Niv, S. (2013). Clinical efficacy and potential mechanisms of neurofeedback. Personality and Individual Differences, 54(6), 676-686. http://dx.doi.org/10.1016/j.paid.2012.11.037

Pallant, J. (2007). SPSS Survival Manual: A Step by Step Guide to Data Analysis Using SPSS for Windows Version 15 (3rd ed.). Milton Keynes, UK: Open University Press.

Porges, S. W. (2007). The polyvagal perspective. Biological Psychology, 74(2), 116-143. http://dx.doi.org/10.1016 lj.biopsycho.2006.06.009

ProComp2 amplifier with BioGraph 5.1 software [Apparatus]. Montreal, Canada: Thought Technology Ltd.

ProComp5 amplifier with BioGraph 5.1 software [Apparatus]. Montreal, Canada: Thought Technology Ltd.

$\mathrm{R}$ Core Team. (2016). R: A language and environment for statistical computing. Vienna, Austria: $\mathrm{R}$ Foundation for Statistical Computing. Retrieved from https://www.rproject.org/

Reid-Chung, A., Thompson, M., \& Thompson, L. (2015). Heart Rate Variability and Traumatic Brain Injury (TBI): Clinical Applications. Biofeedback, 43(1), 27-30. http://dx.doi.org /10.5298/1081-5937-43.1.02

Richards, D. (2011). Prevalence and clinical course of depression: A review. Clinical Psychology Review, 31(7), 1117-1125. http://dx.doi.org/10.1016/j.cpr.2011.07.004

Royal College of Psychiatrists. (2013). Second Round of the National Audit of Psychological Therapies for Anxiety and Depression (NAPT) 2013. London, UK: Healthcare Quality Improvement Partnership.

Sadjadi, S. A., \& Hashemian, P. (2014). Effectiveness of Neurofeedback Therapy in Children with Separation Anxiety Disorder. Journal of Psychiatry, 17, 10000149. http://dx.doi.org/10.4172/Psychiatry.1000149

Schneider, R. L., Arch, J. J., \& Wolitzky-Taylor, K. B. (2015). The state of personalized treatment for anxiety disorders: A systematic review of treatment moderators. Clinical Psychology Review, 38, 39-54. http://dx.doi.org/10.1016 /j.cpr.2015.02.004

Shaffer, F., McCraty, R., \& Zerr, C. L. (2014). A healthy heart is not a metronome: An integrative review of the heart's anatomy and heart rate variability. Frontiers in Psychology, 5, 1040. http://dx.doi.org/10.3389/fpsyg.2014.01040

Simkin, D. R., Thatcher, R. W., \& Lubar, J. (2014). Quantitative EEG and neurofeedback in children and adolescents: Anxiety disorders, depressive disorders, comorbid addiction and attention-deficit/hyperactivity disorder, and brain injury. Child \& Adolescent Psychiatric Clinics, 23(3), 427-464. http://dx.doi.org/10.1016/j.chc.2014.03.001 
Song, H.-S., \& Lehrer, P. M. (2003). The effects of specific respiratory rates on heart rate and heart rate variability. Applied Psychophysiology and Biofeedback, 28(1)M, 13-23. http://dx.doi.org/10.1023/A:1022312815649

Sterman, M. B. (1996). Physiological origins and functional correlates of EEG rhythmic activities: Implications for selfregulation. Biofeedback and Self-regulation, 21(1), 3-33. http://dx.doi.org/10.1007/BF02214147

Stuart, A. (1955). A Test for Homogeneity of the Marginal Distributions in a Two-Way Classification. Biometrika, 42(3/4), 412-416. http://dx.doi.org/10.2307/2333387

Swingle, P. G. (2015). Adding neurotherapy to your practice: Clinician's guide to the ClinicalQ, neurofeedback, and braindriving. New York, NY: Springer International Publishing. http://dx.doi.org/10.1007/978-3-319-15527-2

Szkodny, L. E., Newman, M. G., \& Goldfried, M. R. (2014). Clinical Experiences in Conducting Empirically Supported Treatments for Generalized Anxiety Disorder. Behavior Therapy, 45(1), 7-20. http://dx.doi.org/10.1016 /j.beth.2013.09.009

The Task Force Report. (1996). Heart rate variability: Standards of measurement, physiological interpretation, and clinical use. Task Force of the European Society of Cardiology and the North American Society of Pacing and Electrophysiology. Circulation, 93(5), 1043-1065. http://doi.org/10.1161 /01.CIR.93.5.1043

Thompson, M., \& Thompson, L. (2006). Improving Attention in Adults and Children: Differing Electroencephalography Profiles and Implications for Training. Biofeedback, 34(3), 99105.

Vaschillo, E., Lehrer, P., Rishe, N., \& Konstantinov, M. (2002). Heart rate variability biofeedback as a method for assessing baroreflex function: A preliminary study of resonance in the cardiovascular system. Applied Psychophysiology and Biofeedback, 27(1), 1-27. http://dx.doi.org/10.1023 IA:1014587304314

Vittengl, J. R., Clark, L. A., Dunn, T. W., \& Jarrett, R. B. (2007). Reducing relapse and recurrence in unipolar depression: $A$ comparative meta-analysis of cognitive-behavioral therapy's effects. Journal of Consulting and Clinical Psychology, 75(3), 475-488. http://dx.doi.org/10.1037/0022-006X.75.3.475

Walker, J. E. (2009). Anxiety Associated with Post Traumatic Stress Disorder-The Role of Quantitative Electro- encephalograph in Diagnosis and in Guiding Neurofeedback Training to Remediate the Anxiety. Biofeedback, 37(2), 6770. http://dx.doi.org/10.5298/1081-5937-37.2.67

Walker, J. E. (2013). QEEG-Guided Neurofeedback for Anger/Anger Control Disorder. Journal of Neurotherapy, 17(1), 88-92. http://dx.doi.org/10.1080 /10874208.2012.705767

Walker, J. E., \& Lawson, R. (2013). FP02 Beta Training for DrugResistant Depression-A New Protocol That Usually Reduces Depression and Keeps It Reduced. Journal of Neurotherapy, 17(3), 198-200. http://dx.doi.org/10.1080 /10874208.2013.785784

Waller, G. (2009). Evidence-based treatment and therapist drift. Behaviour Research and Therapy, 47(2), 119-127. http://dx.doi.org/10.1016/j.brat.2008.10.018

Weimer, K., Colloca, L., \& Enck, P. (2015). Placebo effects in psychiatry: Mediators and moderators. The Lancet Psychiatry, 2(3), 246-257. http://dx.doi.org/10.1016/S22150366(14)00092-3

Westra, H. A. (2011). Comparing the predictive capacity of observed in-session resistance to self-reported motivation in cognitive behavioral therapy. Behaviour Research and Therapy, 49(2), 106-113. http://dx.doi.org/10.1016 /j.brat.2010.11.007

Williams, D. P., Cash, C., Rankin, C., Bernardi, A., Koenig, J., \& Thayer, J. F. (2015). Resting heart rate variability predicts self-reported difficulties in emotion regulation: A focus on different facets of emotion regulation. Frontiers in Psychology, 6, 261. http://dx.doi.org/10.3389/fpsyg.2015.00261

Winkelmann, T., Thayer, J. F., Pohlack, S., Nees, F., Grimm, O., \& Flor, H. (2016). Structural brain correlates of heart rate variability in a healthy young adult population. Brain Structure and Function, 222(2), 1061-1068. http://dx.doi.org/10.1007 Is00429-016-1185-1

Yasuma, F., \& Hayano, J.-I. (2004). Respiratory Sinus Arrhythmia: Why Does the Heartbeat Synchronize with Respirator Rhythm? Chest, 125(2), 683-690. http://dx.doi.org /10.1378/chest.125.2.683

Received: November 10, 2016

Accepted: February 12, 2017

Published: March 15, 2017 


\section{Appendix}

\section{Supplemental Table 1}

ASEBA-Defined Minimal Clinically Important Difference

\begin{tabular}{lcccc|cccc}
\hline & \multicolumn{3}{c}{ Anxiety } \\
\cline { 2 - 8 } & Age & $S D$ & Reliability & MCID & $S D$ & Reliability & MCID \\
\cline { 2 - 8 } Boys & $6-11$ & 5.5 & 0.80 & 2.46 & 5.6 & 0.84 & 2.24 \\
& $12-18$ & 5.7 & 0.80 & 2.55 & 5.8 & 0.84 & 2.32 \\
\multirow{3}{*}{ Girls } & $6-11$ & 5.4 & 0.80 & 2.41 & 5.4 & 0.84 & 2.16 \\
& $12-18$ & 5.7 & 0.80 & 2.55 & 5.8 & 0.84 & 2.32 \\
\hline \multirow{2}{*}{ Men } & $18-35$ & 5.3 & 0.86 & 1.98 & 5.9 & 0.86 & 2.21 \\
& $36-59$ & 4.4 & 0.86 & 1.65 & 5.4 & 0.86 & 2.02 \\
\multirow{3}{*}{ Women } & $18-35$ & 5.4 & 0.86 & 2.02 & 5.6 & 0.86 & 2.10 \\
& $36-59$ & 5.0 & 0.86 & 1.87 & 5.8 & 0.86 & 2.17 \\
\hline
\end{tabular}

Note. Standard deviation $(S D)$ and Reliability statistics are from ASEBA's age- and gender-normed population (Achenbach \&

Rescorla, 2001, 2003). MCID $=$ Minimal Clinically Important Difference $=S D(\sqrt{1-\text { Reliability }})$. 